\title{
SIMULTANEOUS X-RAY AND ULTRAVIOLET OBSERVATIONS OF THE SW SEXTANTIS STAR DW URSAE MAJORIS
}

\author{
D. W. Hoard ${ }^{1,2}$, Ting-Ni Lu ${ }^{3}$, Christian Knigge ${ }^{4}$, Lee Homer ${ }^{5}$, Paula Szkody ${ }^{5}$,

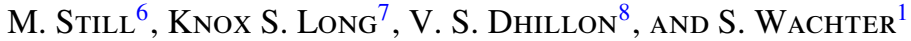 \\ ${ }^{1}$ Spitzer Science Center, California Institute of Technology, MS 220-6, 1200 E. California Blvd., Pasadena, CA 91125, USA \\ 2 Eureka Scientific, Inc., 2452 Delmer Street, Suite 100, Oakland, CA 94602, USA; hoard@ipac.caltech.edu \\ ${ }^{3}$ Institute of Astronomy, National Tsing Hua University, Hsinchu 30013, Taiwan \\ ${ }^{4}$ School of Physics and Astronomy, University of Southampton, Highfield, Southampton SO17 1BJ, UK \\ ${ }^{5}$ Department of Astronomy, University of Washington, Box 351580, Seattle, WA 98195-1580, USA \\ ${ }^{6}$ NASA Ames Research Center, Moffett Field, CA 94035, USA \\ 7 Space Telescope Science Institute, 3700 San Martin Drive, Baltimore, MD 21218, USA \\ ${ }^{8}$ Department of Physics and Astronomy, University of Sheffield, Sheffield S3 7RH, UK \\ Received 2010 June 18; accepted 2010 September 4; published 2010 October 13
}

\begin{abstract}
We present the first pointed X-ray observation of DW Ursae Majoris, a novalike cataclysmic variable (CV) and one of the archetype members of the SW Sextantis class, obtained with the XMM-Newton satellite. These data provide the first detailed look at an SW Sex star in the X-ray regime (with previous X-ray knowledge of the SW Sex stars limited primarily to weak or non-detections in the ROSAT All Sky Survey). It is also one of only a few XMMNewton observations (to date) of any high mass transfer rate novalike CV, and the only one in the evolutionarily important 3-4 hr orbital period range. The observed X-ray spectrum of DW UMa is very soft, with $\sim 95 \%$ of the detected X-ray photons at energies $<2 \mathrm{keV}$. The spectrum can be fit equally well by a one-component cooling flow model, with a temperature range of $0.2-3.5 \mathrm{keV}$, or a two-component, two-temperature thermal plasma model, containing hard $(\sim 5-6 \mathrm{keV})$ and soft $(\sim 0.8 \mathrm{keV})$ components. The X-ray light curve of DW UMa shows a likely partial eclipse, implying X-ray reprocessing in a vertically extended region, and an orbital modulation, implying a structural asymmetry in the X-ray reprocessing site (e.g., it cannot be a uniform corona). We also obtained a simultaneous near-ultraviolet light curve of DW UMa using the Optical Monitor on XMM-Newton. This light curve is similar in appearance to published optical-UV light curves of DW UMa and shows a prominent deep eclipse. Regardless of the exact nature of the X-ray reprocessing site in DW UMa, the lack of a prominent hard X-ray total eclipse and very low fraction of high energy X-rays point to the presence of an optically and geometrically thick accretion disk that obscures the boundary layer and modifies the X-ray spectrum emitted near the white dwarf.
\end{abstract}

Key words: accretion, accretion disks - novae, cataclysmic variables - stars: individual (DW Ursae Majoris) X-rays: binaries - X-rays: stars

Online-only material: color figures

\section{INTRODUCTION}

Cataclysmic variables (CVs) are semi-detached binary stars in which a late main-sequence star loses mass onto a white dwarf (WD) via Roche lobe overflow. In systems containing a nonmagnetic WD, accretion onto the WD then proceeds through a viscous disk (see Warner 1995 for a thorough review of CV types and behavior). In the late 20th century, three CVs (SW Sex, DW $\mathrm{UMa}$, and V1315 Aql) were proposed as the founding members of a new CV subclass, the "SW Sex Stars" (Honeycutt et al. 1986; Szkody \& Piché 1990; Thorstensen et al. 1991). These CVs shared a number of defining observational characteristics, such as the following.

1. Spectra that exhibit high levels of excitation (including He II $\lambda 4686$ emission that is often comparable in strength to $\mathrm{H} \beta$ ).

2. Zero-crossings of their emission line radial velocities with pronounced phase offsets relative to their eclipse ephemerides (implying a non-uniform distribution of emitting regions in the disk).

3. Absorption features that appear in their Balmer and He I emission line cores, often extending below the continuum and typically manifesting around photometric phase $\phi=$
0.5 (i.e., the superior conjunction of the secondary star, opposite the eclipse).

Additional CVs matching the observational characteristics of the first three SW Sex stars were subsequently identified. ${ }^{9}$

The presence of deep eclipses (i.e., high inclination) was originally included as a defining characteristic of the SW Sex stars, despite the discomfiting status of this property as a purely coincidental result of the system orientation relative to the plane of the sky. Subsequently, however, a substantial fraction of SW Sex stars with moderate or low inclinations were identified (e.g., WX Ari, Beuermann et al. 1992; Hellier et al. 1994; Rodríguez-Gil et al. 2000; V442 Oph, Hoard et al. 2000; BO Cet and AH Men, Rodríguez-Gil et al. 2007). High inclination produces a selection bias by causing a CV to be more likely to be studied in detail (due to the presence of "interesting" eclipses) and, therefore, more likely to be identified as an SW Sex star through observation of the diagnostic characteristics listed above. A definitive explanation for the observed characteristics and behavior of the SW Sex stars has yet to be formulated, despite substantial work in this area (see Hoard et al. 2003 for a

\footnotetext{
9 See the Big List of SW Sex Stars at

http://www.dwhoard.com/cvtools/swsex/biglist.html.
} 
detailed summary of SW Sex star observational characteristics and models).

In a broader sense, the SW Sex stars are novalike CVs (although not all novalike CVs are SW Sex stars). Novalikes are characterized by an approximately steady, high rate of mass transfer that quenches the thermal-tidal disk instability mechanism responsible for dwarf nova outbursts in other CVs (Osaki 1996; Buat-Ménard et al. 2001; Lasota 2001). Some novalikes occasionally and unpredictably undergo so-called low states lasting from weeks up to several years during which their optical brightness drops by up to $\sim 5 \mathrm{mag}$, presumably due to a not-yet fully understood mechanism that throttles the mass transfer rate and causes a significant decrease in accretion-generated luminosity (Honeycutt \& Kafka 2004). DW UMa experienced low states in 1990-1993 and 1998-1999 (Honeycutt \& Kafka 2004; Knigge et al. 2000).

In the orbital period census of CVs, almost all of the SW Sex stars are found on the long-period edge of the $\approx 2-3 \mathrm{hr}$ orbital period gap (Rappaport et al. 1983; Spruit \& Ritter 1983), poised to transition into the gap as their orbital periods decrease over time, following the trend of the secular evolution of CVs. The period gap itself is characterized by a different observed distribution of CV types, notably a dearth of non-magnetic systems. The "missing" systems are presumed to be CVs that have gone into "hibernation" and become too faint to easily detect. This hibernation, in turn, is presumed to result from the cessation of accretion due to a change in the dominant orbital angular momentum loss mechanism that results in the secondary star losing contact with its Roche lobe. By the time a CV evolves to an orbital period near $2 \mathrm{hr}$, its secondary star comes back into contact with its Roche lobe, mass transfer and accretion resume, and the hibernating CV becomes bright again. The period gap is more or less universally agreed to be both crucially important to an understanding of the evolution of CVs (e.g., Willems et al. 2005) and largely inexplicable in the context of recent understanding of the magnetic properties (Kafka \& Honeycutt 2006) and angular momentum loss rates (Andronov et al. 2003) of low-mass stars. Consequently, understanding the SW Sex stars may be crucially important to understanding the evolution of CVs as a class.

The archetype SW Sex star DW UMa was first studied in detail by Shafter et al. (1988, also see Zhang 1988), who obtained extensive optical photometric and spectroscopic data. More recent observations have extended into the mid- to farultraviolet (FUV, e.g., Knigge et al. 2000, 2004; Hoard et al. 2003). DW UMa has an orbital period of $3.28 \mathrm{hr}$ (Stanishev et al. 2004) and a high inclination $\left(i=82^{\circ} \pm 4^{\circ}\right.$; AraujoBetancor et al. 2003) that produces deep eclipses (e.g., see the optical and UV light curves in Shafter et al. 1988 and Knigge et al. 2004, respectively). By comparing UV spectra obtained during high and low states of DW UMa, Knigge et al. (2000) and Knigge et al. (2004) showed that the hot $(T \approx 50,000 \mathrm{~K})$ WD in DW UMa is completely obscured by the optically thick accretion disk rim during the high state. They postulated that the presence of such a disk in other CVs might be an important contributing factor to the origin of the SW Sex characteristics. Notably, given that the hottest part of the CV (i.e., the WD and inner disk) is hidden in DW UMa, we should expect that the observed high energy (UV, X-ray) flux from this CV (and similar SW Sex stars) should be both weak and deeply eclipsed.

This expectation was already confirmed by the observation of deep UV eclipses in DW UMa (Knigge et al. 2004) and is also generally borne out by the relative weakness of X-ray emission in the $0.24-2.0 \mathrm{keV}$ range from SW Sex stars in the ROSAT All Sky Survey (RASS; White et al. 2000). Nonetheless, of the historically well-known SW Sex stars, DW UMa had the highest RASS X-ray count rate $\left(1.4 \pm 0.2 \mathrm{ct} \mathrm{s}^{-1}\right.$ compared to $0.004 \pm 0.001 \mathrm{ct} \mathrm{s}^{-1}$ for WX Ari, $0.0062 \pm 0.0006 \mathrm{ct} \mathrm{s}^{-1}$ for PX And, and non-detections for SW Sex, V1315 Aql, V1776 Cyg, BH Lyn, and V442 Oph), suggesting it as the most suitable target for future exploration in the X-ray regime. While approximately a dozen dwarf novae have been observed with XMM-Newton (e.g., see Pandel et al. 2003, 2005; Wheatley \& West 2003; Hilton et al. 2007; Nucita et al. 2009b), only three novalike CVs have been observed: UX UMa (Pratt et al. 2004), SDSS093214.82+495054.7 (Hilton et al. 2009), and now DW UMa. Little is known about SDSS0932: it likely has a very long orbital period, in excess of $10 \mathrm{hr}$, and only an upper limit Xray flux of $0.014 \mathrm{ct} \mathrm{s}^{-1}$ from XMM-Newton. The XMM-Newton observation of UX UMa is discussed in Section 3.

We now present the first pointed X-ray observation of DW UMa, obtained with the XMM-Newton satellite (Jansen et al. 2001). These data provide the first detailed look at an archetype SW Sex star in the X-ray regime (previous X-ray knowledge of the SW Sex stars being limited primarily to the aforementioned weak- and non-detections in the RASS), as well as being one of only a few XMM-Newton observations (to date) of any high mass transfer rate novalike $\mathrm{CV}$, and the only one in the evolutionarily important $\approx 3-4 \mathrm{hr}$ orbital period range that is located just above the period gap.

\section{OBSERVATIONS AND ANALYSIS}

We observed DW UMa with XMM-Newton on 2003 October 18 UT (JD 2452930.5) for approximately 25,000 s (see Table 1). $\mathrm{X}$-ray spectra and light curves were extracted from all three cameras (PN, MOS1, and MOS2) of the European Photon Imaging Camera (EPIC) data, starting from the XMM-Newton Science Analysis Software (SAS), version 9.0.0, and using HEASOFT, version 6.4. The Observation Data Files were reprocessed by running the pipeline modules emchain and epchain for the MOS and PN cameras, respectively. Periods of high background flares (photon count rates $>0.5 \mathrm{ct} \mathrm{s}^{-1}$ and $>3.5 \mathrm{ct} \mathrm{s}^{-1}$ for MOS and PN, respectively) were eliminated from the X-ray data. We also excluded event grades higher than 12 for the MOS cameras and higher than 4 for the PN camera. The final effective exposure times were reduced to $16.7,18.2$, and $17.2 \mathrm{ks}$ for the MOS1, MOS2, and PN cameras, respectively.

Because DW UMa is neither especially bright (which can cause scattered light problems) nor in a crowded field, we used the SAS default pipeline-extracted Optical Monitor (OM; Mason et al. 2001) light curve, which has a time resolution of 10 s per measurement. The near-ultraviolet $U V W 2$ filter (effective wavelength $2905 \AA$ with FWHM $620 \AA$ ) was used for the OM observation. Although the Reflection Grating Spectrometer was active, there was no measurable detection of the dispersed spectrum.

We also acquired 10 out-of-eclipse, ground-based CCD images of DW UMa in each of the $B$ and $V$ bands ${ }^{10}$ during JD 2452930.96-2452931.00, immediately following the XMMNewton observation (which ended at JD 2452930.81). These data were calibrated by comparison with simultaneous observations of the secondary photometric standards of Henden \& Honeycutt (1995) to give mean brightnesses for DW UMa of $B=14.51 \pm 0.01$ and $V=14.17 \pm 0.01$. This places DW UMa

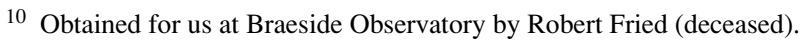


Table 1

Log of XMM-Newton Observations 0142970101

\begin{tabular}{|c|c|c|c|c|c|c|}
\hline Instrument & UT Start & UT Stop & Duration (s) & Mode & Data Mode & Filter \\
\hline MOS2 & 2003-10-18T00:23:58 & $07: 25: 05$ & 25267 & PrimePartialW2 & Imaging & Thin1 \\
\hline $\mathrm{OM}^{\mathrm{a}}$ & 2003-10-18T00:32:14 & 07:27:02 & 21795 & Image & Imaging and fast & $U V W 2$ \\
\hline
\end{tabular}

Note. ${ }^{\text {a }}$ The Optical Monitor observation was split into five exposures, each of duration $\approx 4359$ s, separated by $\approx 5-35$ minutes.

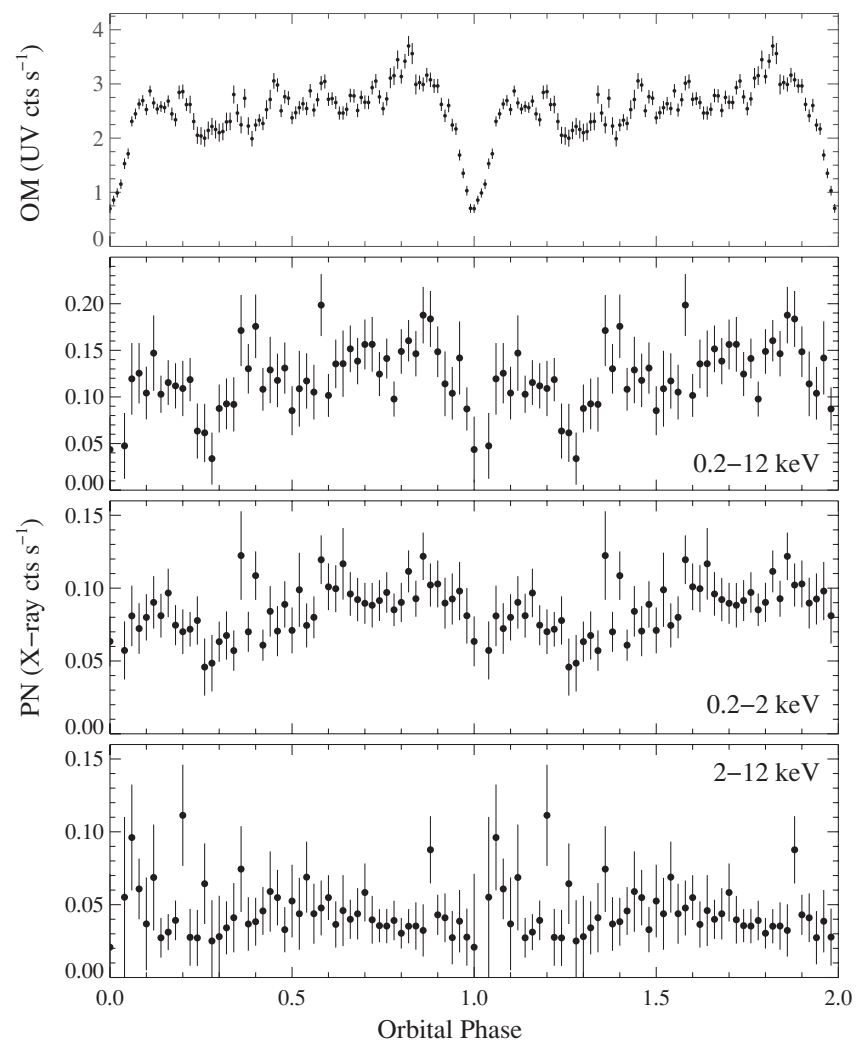

Figure 1. Orbital-phase-binned light curves of DW UMa. From top to bottom the panels are XMM-Newton Optical Monitor $U V W 2$ filter and XMM-Newton PN X-ray in the energy ranges $0.2-12 \mathrm{keV}, 0.2-2 \mathrm{keV}$, and $2-12 \mathrm{keV}$. (Note the change in scale of the full energy range $\mathrm{X}$-ray light curve compared to the two energy-binned X-ray light curves.) In all panels, the data are repeated over two orbital cycles.

securely in its normal high state (e.g., Honeycutt \& Kafka 2004) during our XMM-Newton observations.

\subsection{X-ray and Optical Light Curves}

Figure 1 shows our simultaneous XMM-Newton PN (X-ray) and OM (near-UV) light curves of DW UMa. Each light curve consists of approximately two orbital periods of phase coverage that were folded on the orbital period of Stanishev et al. (2004). An offset of $+1650 \mathrm{~s}$ was applied to the times of the OM data (which were recorded as seconds after 1998.0 UTC in the Terrestrial Time system) so that the center of the observed eclipse coincided exactly with the nearest-in-time zero point $(\phi=0.0)$ of the Stanishev et al. (2004) ephemeris. The same time offset was applied to the PN data. The folded data were averaged into $100(\mathrm{OM})$ or $50(\mathrm{PN})$ phase bins per orbit.

The OM light curve is, in many ways, similar in appearance to published optical and ultraviolet light curves of DW UMa (e.g.,
Shafter et al. 1988; Knigge et al. 2004). For example, it shows a deep, relatively broad eclipse, and a low level of stochastic variability outside of eclipse (i.e., "flickering"). The hump at $\phi \approx 0.75-0.9$ corresponds to the bright spot at the impact site of the accretion stream with the disk edge. This feature is not always seen in light curves of DW UMa, but it is also not without precedent (e.g., a similar pre-eclipse hump is present in several of the many DW UMa light curves shown in Stanishev et al. 2004).

The X-ray light curves were constructed from the summed, flare-filtered, background-subtracted count rates of DW UMa from the EPIC-PN camera. The MOS1 and MOS2 cameras yielded count rates that were a factor of $\gtrsim 3$ smaller than the PN and were not utilized for the timing analysis. The event list data were first binned into intervals of $50 \mathrm{~s}$ and converted to count rates before averaging into the 50 orbital phase bins. Light curves were constructed for several energy ranges: $0.2-12 \mathrm{keV}$ (full), 0.2-2 keV (soft), and 2-12 keV (hard). The full energy range $\mathrm{X}$-ray light curve echoes many of the features of the OM light curve. For example, it shows a dip at $\phi \approx 0.3$, followed by a gradual rise to $\phi \approx 0.9$. The presence of several bright and faint points (relative to the overall trend) in the X-ray light curve at $\phi \approx 0.35-0.6$ matches the relatively high level of short timescale variability in the OM light curve in this phase range.

Figure 2 presents the $\mathrm{OM}$ and $\mathrm{PN}$ light curve data in a somewhat different fashion than shown in Figure 1. The OM data points are phased onto the orbital ephemeris of DW UMa, as described above, but have not been averaged into phase bins; instead, they are the original $10 \mathrm{~s}$ binned points described in Section 2. The PN data have been phase binned as described above, but data from the first and second observed orbital cycles of DW UMa were kept separate. These light curves indicate that there is generally good agreement in the brightness of DW UMa - in both the near-UV and X-rays - at the same phase from orbit to orbit. The caveat to this is that we have only a single orbit of coverage in the phase range $\phi \approx 0.2-0.3$, so cannot be certain that the behavior in this range-which corresponds to a dip in both the OM and PN light curves-persists from orbit to orbit. However, the behavior of the data from the second orbit directly adjacent to the ends of this phase range is consistent with that of the first orbit; so we suspect that the $0.2-0.3$ phase range is likely showing true orbital variability.

There is a narrow dip in the X-ray light curve at $\phi \approx 0.0$ that is highly suggestive of an X-ray eclipse. The minimum of the dip corresponds to an $\approx 60 \%$ reduction in X-ray count rate, which indicates that it is a partial, not total, eclipse. We note that the range of observation times corresponding to this orbital phase range suffered from significant rejection due to background flares. For example, the phase bin centered at $\phi=0.02$ is empty. However, the three bins that most clearly show the possible eclipse, $\phi=0.98,0.00$, and 0.04, contain five, five, and four data points, respectively. This is comparable to the six and five 


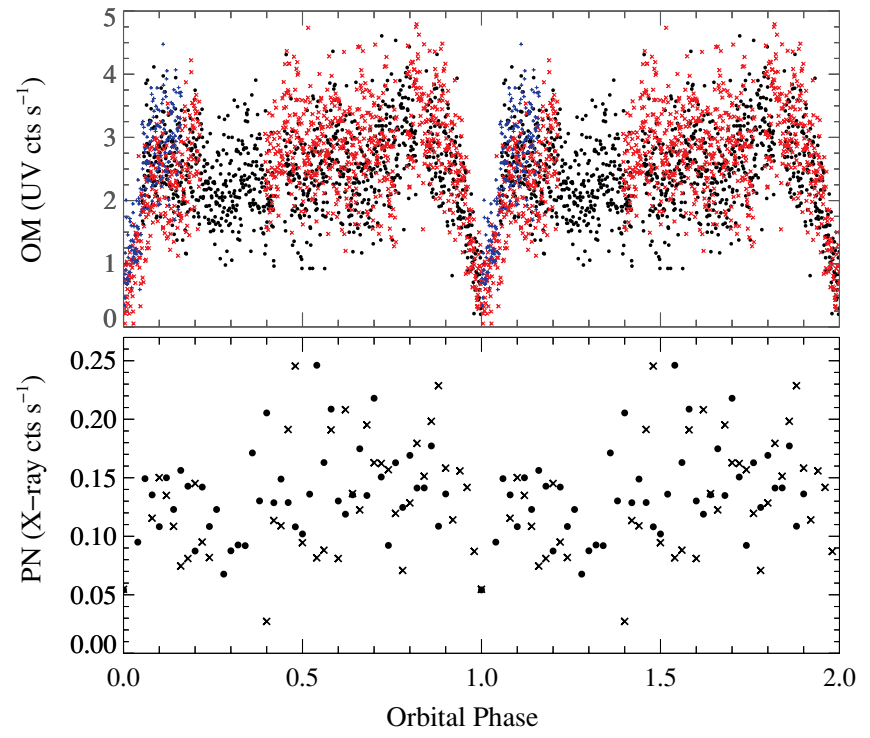

Figure 2. Light curves of DW UMa, as in Figure 1, but showing the unbinned data from the OM (top) and the PN (full energy range; bottom). In both panels, the first DW UMa orbital cycle is shown as filled (black) circles, while the second orbital cycle is shown as (red in the online version) $\times$ symbols. The top panel also shows brief coverage of the start of a third orbital cycle, observed by the OM only, as (blue in the online version) + symbols. In both panels, the data are repeated over two orbital cycles.

(A color version of this figure is available in the online journal.)

data points averaged into each of the adjacent bins on the preeclipse side, at $\phi=0.94$ and 0.96 , respectively, as well as the five and eight points averaged into the two adjacent bins on the post-eclipse side, at $\phi=0.06$ and 0.08 , respectively. Thus, this feature, putatively a partial eclipse of the X-rays in DW UMa, appears to be real.

A dip in the full and soft X-ray light curves, comparable in duration and depth to the eclipse dip, is present at $\phi \approx 0.3$. As discussed above, this X-ray dip corresponds to a shallow dip in the OM light curve at the same orbital phase. Interestingly, although the overall impression of the X-ray eclipse is that it is narrower than the near-UV/optical eclipse (which is consistent with observations of X-ray eclipses in other CVs; see discussion in Section 3), this appears to result primarily from the visual effect of the two lowest points. A direct comparison of the Xray and near-UV eclipse profiles (see Figure 3) reveals that this situation is somewhat more ambiguous. The evolution of the $\mathrm{X}$-ray light curve through eclipse is actually quite similar in appearance to that of the near-UV light curve, in terms of the eclipse profile shape, width, and depth relative to the adjacent continuum.

The features of the full energy range X-ray light curve (Figure 1) are mirrored in the soft X-ray light curve, which is not surprising considering that approximately $95 \%$ of the total $\mathrm{X}$-ray counts emerge in the $0.2-2 \mathrm{keV}$ energy range (e.g., see Figure 4). The hard X-ray light curve is overall noisier in keeping with the much smaller count rate in this energy range. There is some indication for a shallow, sinusoidal variation in the light curve, but with a phase offset compared to the orbital variation in the soft $\mathrm{X}$-ray light curve (e.g., maximum at $\phi \approx 0.5$ in hard $\mathrm{X}$-rays versus $\phi \approx 0.9$ in soft $\mathrm{X}$-rays). It is also consistent with an essentially flat X-ray count rate within the uncertainties of almost all of the binned points. There is no evidence for an eclipse; in fact, the most interesting feature of the hard X-ray light curve is a sequence of points just after the nominal eclipse

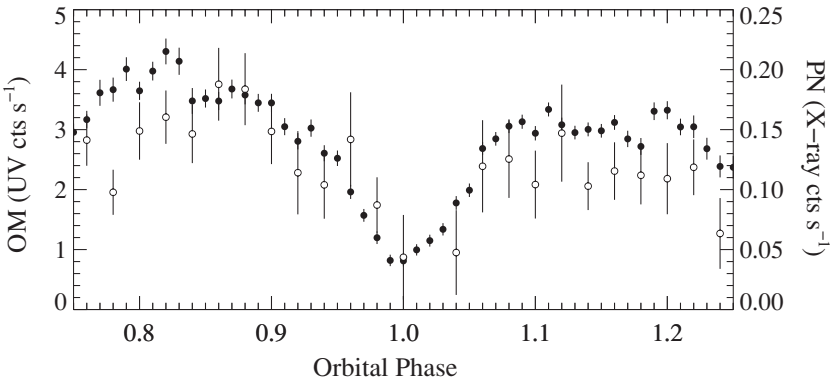

Figure 3. Expanded view of the eclipse profile from the OM data (filled circles and left vertical axis) superimposed with the PN data (full energy range; unfilled circles and right vertical axis) from Figure 1.

phase that imply a slightly higher X-ray count rate. If real, this feature is short-lived $(\Delta \phi \approx 0.1)$; however, since the averaged points from $\phi=0.98$ to $\phi=0.06$ contain only one or two contributing points in their phase bins, this feature might not be reliable.

The most interesting aspect of the X-ray light curve of DW UMa is the presence of a possible partial eclipse; as noted in Section 1, we should expect the X-rays in DW UMa to be deeply eclipsed. Because of the low count rate (and, hence, low signalto-noise ratio, $\mathrm{S} / \mathrm{N}$ ) and poor time sampling of the DW UMa $\mathrm{X}$-ray light curve, it is difficult to make a meaningful comparison with the X-ray light curves of other CVs; for example, the flatbottomed total eclipses seen in the XMM-Newton observation of the dwarf novae HT Cas (Nucita et al. 2009b) and OY Car (Ramsay et al. 2001), or the partial X-ray eclipse observed with Suzaku in the dwarf nova V893 Sco (Mukai et al. 2009). The best comparison might be made with the X-ray eclipse seen in $X M M$ Newton observations of the longer-period $\left(P_{\text {orb }}=4.72 \mathrm{hr}\right)$ novalike CV UX UMa (Pratt et al. 2004); however, we note that in the case of UX UMa, the eclipse is observed in only the hard X-ray light curve and not the soft, whereas the situation is reversed for DW UMa.

One feature of note that does appear to be consistent with the X-ray eclipse in UX UMa is the apparent asymmetry of the DW UMa eclipse profile, which is centered at an orbital phase slightly past $\phi=0.0$. To some extent, this mirrors the asymmetry of the optical-UV eclipse profile in DW UMa (in which the eclipse ingress is steeper and shorter than the eclipse egress; see Figure 3 and also Figure 1 in Shafter et al. 1988), whereas the optical-UV eclipse profile of UX UMa is much more symmetric (e.g., see Figures 1 and 2 in Knigge et al. 1998).

\subsection{X-ray Spectrum}

The background-subtracted EPIC X-ray spectrum of DW $\mathrm{UMa}$, binned to $15 \mathrm{ct} \mathrm{s}^{-1} \mathrm{keV}^{-1}$, is shown in Figure 4. The MOS1 and MOS2 spectra are nearly identical, so only the MOS1 spectrum is shown in the figure. There are some emission line features in the spectrum, notably $\mathrm{Mg}$ XII at $1.47 \mathrm{keV}$, as well as possible weak detections of N vII at $0.5 \mathrm{keV}$ and the Fe $\mathrm{K} \alpha$ line at $6.7 \mathrm{keV}$. Spectral model fits were performed jointly to the $\mathrm{PN}, \mathrm{MOS} 1$, and MOS2 spectra. The results of the model fits are listed in Table 2 and discussed below. An abundance of $Z=0.5$ (Knigge et al. 2000) was used in all models.

One-component, single temperature, optically thin thermal plasma models (e.g., MEKAL and APEC) produced poor fits to the DW UMa X-ray spectrum (with reduced chi-squared values $\chi_{v}^{2}>2$ ). However, a one-component cooling flow model (MKCFLOW) with a temperature distribution ranging from $0.2 \mathrm{keV}$ to $3.5 \mathrm{keV}$ produced an improved fit to the data, with 
Table 2

X-ray Spectrum Models

\begin{tabular}{lccccc}
\hline \hline \multicolumn{1}{c}{ Model $^{\mathrm{a}}$} & $\begin{array}{c}N_{\mathrm{H}}^{\mathrm{b}} \\
\left(10^{19} \mathrm{~cm}^{-2}\right)\end{array}$ & $\begin{array}{c}k T \text { (hard) } \\
(\mathrm{keV})\end{array}$ & $\begin{array}{c}k T \text { (soft) } \\
(\mathrm{keV})\end{array}$ & $\chi_{v}^{2}$, dof & $\begin{array}{c}f_{0.2-10 \mathrm{keV}} \\
\left(10^{-14} \mathrm{erg} \mathrm{s}^{-1} \mathrm{~cm}^{-2}\right)\end{array}$ \\
\hline MKCFLOW & $32 \pm 8$ & $3.5^{\mathrm{c}}$ & $0.2^{\mathrm{c}}$ & $1.42,90$ & 22.7 \\
MEKAL+MEKAL & 6 & $5.4 \pm 1.4$ & $0.77 \pm 0.04$ & $1.41,91$ & 24.7 \\
APEC+APEC & 6 & $5.9 \pm 1.8$ & $0.78 \pm 0.03$ & $1.41,91$ & 24.4 \\
\hline
\end{tabular}

Notes.

${ }^{a}$ MKCFLOW: cooling flow (Mushotzky \& Szymkowiak 1988); MEKAL: thermal plasma (Arnaud \& Rothenflug 1985; Mewe et al. 1985, 1986; Arnaud \& Raymond 1992; Kaastra 1992; Liedahl et al. 1995); APEC: thermal plasma (Smith et al. 2000, 2001a, 2001b).

${ }^{\mathrm{b}} N_{\mathrm{H}}$ was fixed at $6 \times 10^{19} \mathrm{~cm}^{-2}$ for all MEKAL and APEC models.

${ }^{\mathrm{c}}$ Upper and lower temperature limits for the cooling flow model.

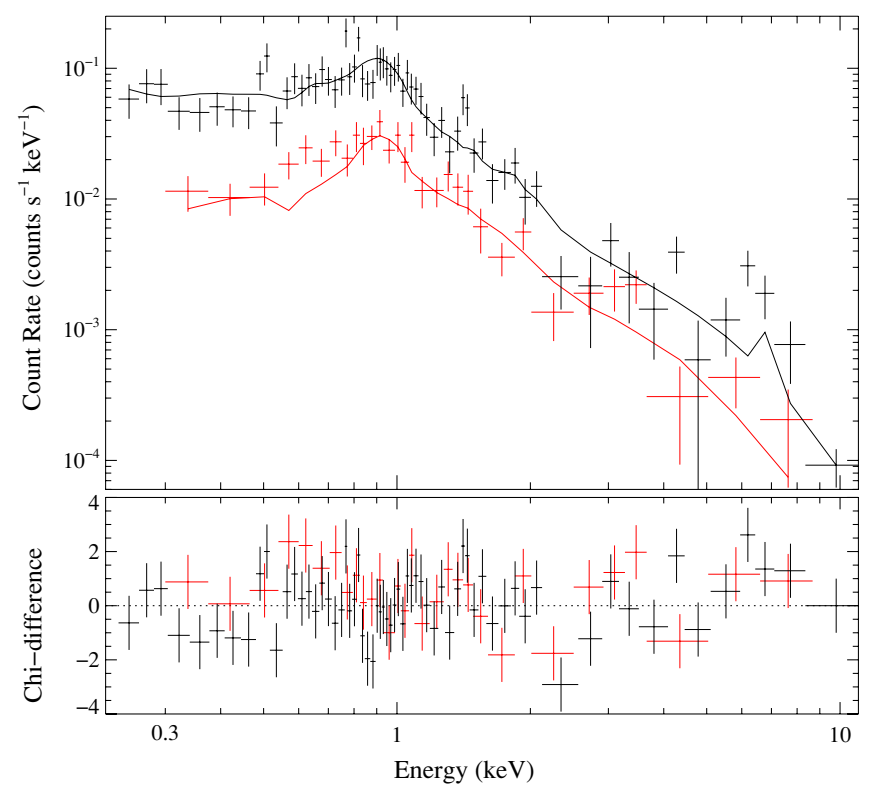

Figure 4. Top: model fit to the total XMM-Newton EPIC-PN (upper curve) and EPIC-MOS1 (lower curve with dark points; red in the online version) observations of DW UMa. (The MOS2 spectrum is indistinguishable from the MOS1 spectrum, so is omitted for clarity.) The model utilizes a two-component twotemperature thermal plasma (MEKAL+MEKAL) model. Bottom: fit residuals shown as a chi-difference plot.

(A color version of this figure is available in the online journal.)

$\chi_{v}^{2}=1.42$. The presence of a variety of emission lines in high $\mathrm{S} / \mathrm{N}$, high-resolution $\mathrm{X}$-ray spectra of CVs, suggests that a multi-temperature model might be appropriate to reproduce the observations. For example, Pandel et al. (2005) found that the MKCFLOW model best reproduced the XMM-Newton spectra for most of the CVs in their sample of 10 dwarf novae (by comparison, three- or four-component thermal plasma models were required to achieve a comparable fit). In addition, Mukai et al. (2003) found that a cooling flow model produced the best match to their Chandra X-ray spectra of four CVs: the dwarf novae U Gem and SS Cyg, the old nova V603 Aql, and the weakly magnetic CV (a so-called intermediate polar) EX Hya. Interestingly, EX Hya has been suggested as a possible SW Sex star (Hellier et al. 2000), especially in the context of the magnetic model for the SW Sex phenomenon (RodríguezGil et al. 2001, 2009; Hoard et al. 2003), since it shows some SW Sex-like characteristics during its outbursts. Both Mukai et al. (2003) and Luna et al. (2010) have noted that its X-ray spectrum shows similarities to both magnetic and non-magnetic systems (see Section 3 for additional discussion of EX Hya and the magnetic model for SW Sex stars).
Another commonly used approach to fitting the X-ray spectra of CVs, especially in the case of low $\mathrm{S} / \mathrm{N}$ data that cannot adequately constrain many model components and/or temperatures, is to utilize two model components with distinctly different temperatures to separately reproduce the hard and soft portions of the spectra (e.g., Nucita et al. 2009a; Pratt et al. 2004). This approach should not necessarily be taken to imply that there are two discrete $\mathrm{X}$-ray emitting regions; instead, it is merely a simplified model that serves as a proxy for a presumed more complex X-ray source that cannot be adequately constrained by the available data. Consequently, we also tried two-component, two temperature models consisting of MEKAL+MEKAL and APEC+APEC thermal plasma components.

In both cases, the best fit was achieved for a soft component with a temperature of $\approx 0.8 \mathrm{keV}$, which produces the blend of lines around $1 \mathrm{keV}$, and a hard component with a temperature of $\approx 5-6 \mathrm{keV}$ that produces the high energy continuum (and also yields the $\mathrm{Fe}$ emission that is weakly detected as the low $\mathrm{S} / \mathrm{N}$ flux excess at $\sim 6.7 \mathrm{keV}$ in our spectrum). The $\chi_{v}^{2}$ value was 1.41 for both models, comparable to that achieved for the one-component cooling flow model described above. We note that these two models (MEKAL and APEC) are generally of the same type (i.e., optically thin thermal plasma) and differ in detail only (e.g., specific atomic data utilized). The consistency between the model-fit parameters in both cases demonstrates the level at which the DW UMa X-ray spectrum is able to constrain the models: the general form of the model (optically thin thermal plasma) reproduces the observed X-ray spectrum well, but the smaller differences between the model assumptions do not significantly affect the quality of the fit.

For the thermal plasma models, the hydrogen column density was fixed at the Galactic average for the location of DW UMa, $N_{\mathrm{H}}=6 \times 10^{19} \mathrm{~cm}^{-2}$. Column density was a free parameter in the cooling flow model, which preferred a higher value of $N_{\mathrm{H}} \approx 32 \times 10^{19} \mathrm{~cm}^{-2}$, but with a relatively large uncertainty of $8 \times 10^{19} \mathrm{~cm}^{-2}$. All of the models yield consistent values for the corresponding $\mathrm{X}$-ray flux in the $0.2-10 \mathrm{keV}$ range, $f_{\mathrm{X}} \sim 2.3-2.4 \times 10^{-13} \mathrm{erg} \mathrm{s}^{-1} \mathrm{~cm}^{-2}$.

We show the MEKAL+MEKAL model overplotted on the data in Figure 4; the other two models are essentially indistinguishable from the plotted model. None of the models reproduce the prominent $\mathrm{Mg}$ XII emission line at $1.47 \mathrm{keV}$. The presence of the $\mathrm{Mg}$ emission line in the observed spectrum could indicate that the abundance of Mg in DW UMa is larger than the $Z=0.5$ used for the model. Pandel et al. (2005), for example, found $\mathrm{Mg}$ abundances of $>0.5$ for 9 out of $10 \mathrm{CVs}$ observed by XMMNewton, even in cases where the abundances of other metals (e.g., C, N, O, Ne, Si, and S) were sub-solar. We experimented with refitting the thermal plasma model using metal abundances 
up to solar, but did not achieve a significantly improved match either overall or specifically to the emission features. Disregarding the possibility of unrealistically high metal abundances, this leaves open the possibility that the true emission source in DW UMa is more complex than either of the relatively simple model forms (thermal plasma and cooling flow) used here.

Overall, allowing for differences in $\mathrm{S} / \mathrm{N}$, the X-ray spectrum of DW UMa is similar in appearance to the typical spectra of CVs observed with XMM-Newton (e.g., see Figure 1 in Pandel et al. 2005; also see the comparably faint XMM-Newton spectrum of the $P_{\text {orb }} \approx 2 \mathrm{hr}$ dwarf nova MACHO 104.20906.960 in Nucita et al. 2009a). The XMM-Newton X-ray spectrum of the novalike CV UX UMa was fit by Pratt et al. (2004) with a two component model in which the hard component had $k T \approx 5.7 \mathrm{keV}$, comparable to the hard component in our twocomponent thermal plasma model fits for DW UMa.

\subsection{X-ray Luminosity and Accretion Rate}

The (unabsorbed) model $0.2-10 \mathrm{keV}$ X-ray flux for DW UMa, $f_{\mathrm{X}} \approx 2.4 \pm 0.1 \times 10^{-13} \mathrm{erg} \mathrm{s}^{-1} \mathrm{~cm}^{-2}$ (see Table 2), implies an X-ray luminosity of $L_{\mathrm{X}} \sim 2 \times 10^{31}(d \text { [pc]/750) })^{2} \mathrm{erg} \mathrm{s}^{-1}$, where $d=750$ pc was chosen to be midway between the two distance estimates to DW UMa determined by Araujo-Betancor et al. (2003) (i.e., $590 \pm 100 \mathrm{pc}$ or $930 \pm 160 \mathrm{pc}$ ). This distance value is likely to be uncertain by $\approx 25 \%$ or more.

The total accretion-generated luminosity in a CV, $L_{\mathrm{acc}}$, is given by

$$
L_{\mathrm{acc}}=\frac{G \dot{M} M_{\mathrm{wd}}}{R_{\mathrm{wd}}},
$$

where $G$ is the gravitation constant, $\dot{M}$ is the mass transfer rate through the disk onto the WD, and $M_{\mathrm{wd}}$ and $R_{\mathrm{wd}}$ are the mass and radius of the $\mathrm{WD}$, respectively. It is generally assumed that the luminosity of the accretion disk, $L_{\text {disk }}$, constitutes $\frac{1}{2} L_{\text {acc }}$, with the remainder of the accretion luminosity accounted for (i.e., radiated) at the boundary layer (i.e., the region of interaction between the inner edge of the accretion disk and the WD). Simplistically, the luminosity of the boundary layer is then $L_{\mathrm{BL}}=L_{\mathrm{disk}}=\frac{1}{2} L_{\mathrm{acc}}$. However, in the presence of a rotating $\mathrm{WD}, L_{\mathrm{BL}}=\frac{1}{4} L_{\mathrm{disk}}=\frac{1}{8} L_{\mathrm{acc}}$ is typically more appropriate, with the "missing" energy having gone into spinning up the accreting WD (Popham \& Narayan 1995). Thus, using the WD mass and radius from Araujo-Betancor et al. (2003) and assuming that $L_{\mathrm{X}} \approx L_{\mathrm{BL}}$, we obtain $\dot{M} \sim 10^{-11} M_{\odot} \mathrm{yr}^{-1}$ for DW UMa. The X-ray luminosity of DW UMa is not atypical for a novalike or dwarf nova in outburst (e.g., $L_{X}=1.3 \times 10^{31}$, $3 \times 10^{31}$, and $9 \times 10^{30} \mathrm{erg} \mathrm{s}^{-1}$ for UX UMa, IX Vel, and Z Cam, respectively; Pratt et al. 2004; Baskill et al. 2005; Baskill et al. 2001). However, data from longer wavelengths imply a mass transfer rate two or more orders of magnitude higher for both DW UMa itself (Linnell et al. 2008) and novalike CVs in general (Ballouz \& Sion 2009). This issue is discussed further below.

\section{DISCUSSION}

The troubling aspect of the partial X-ray eclipse detection in DW UMa is that if the X-rays do originate in the boundary layer region near the WD, then we should expect them to be deeply eclipsed in a high-inclination system like DW UMa. At an even more basic level, in a CV like DW UMa, which has been shown to contain (during the high state) a geometrically thick, self-occulting accretion disk (Knigge et al. 2000, 2004), we should probably not expect to see any X-rays at all.

A solution to this apparent paradox is not difficult to reach: we are seeing X-rays originally emitted with a hard spectrum at the boundary layer, but reprocessed into soft X-rays and re-emitted (e.g., via thermalization of plasma in the disk) or scattered above the disk (e.g., via Compton scattering). This explains both the soft X-ray spectrum of DW UMa (with $~ 95 \%$ of the detected $\mathrm{X}$-rays at energies $<2 \mathrm{keV}$ ) and the lack of a total eclipse (if the reprocessing/re-emission site is partially visible above the projected height of the secondary star against the disk). With respect to the latter condition, at an inclination of $82^{\circ}$ and mass ratio of 0.4 (Araujo-Betancor et al. 2003), some X-ray emission would have to be visible during eclipse at a height of $\gtrsim 0.3 L_{1}\left(\sim 0.25 R_{\odot} ; L_{1}\right.$ is the distance from the WD to the inner Lagrangian point) above/below the disk mid-plane (see Equation (7) and Figure 13 in Dhillon et al. 1991).

In this context, the value of $\dot{M}$ calculated in Section 2.3 is certainly a lower limit to the true accretion rate in DW UMa, since we are viewing only the fraction of reprocessed X-rays visible above the near-edge-on, thick accretion disk. From the empirical results of Baskill et al. (2005), we expect an increase of one to two orders of magnitude in both $L_{\mathrm{X}}$ and $\dot{M}$ if DW UMa was viewed face-on $\left(i=0^{\circ}\right)$ instead of near edge-on $\left(i \approx 82^{\circ}\right.$; Araujo-Betancor et al. 2003). Similarly, the empirical relation between inclination and $V$-band absolute magnitude found by Warner (1986) for nova remnants implies a luminosity increase by a factor of $\gtrsim 10$ if DW UMa was viewed face-on, assuming that the X-ray luminosity is affected by viewing geometry in the same way as the optical luminosity. Thus, the true intrinsic X-ray luminosity in DW UMa is possibly $L_{\mathrm{X} \text {,true }} \gtrsim 10^{33} \mathrm{erg} \mathrm{s}^{-1}$, which is brighter than the most X-ray luminous low-inclination CVs (Baskill et al. 2005) and corresponds to a mass accretion rate of $\dot{M} \gtrsim 10^{-9} M_{\odot} \mathrm{yr}^{-1}$. This value is consistent with the $\dot{M} \sim 10^{-9} M_{\odot} \mathrm{yr}^{-1}$ estimated by Townsley \& Bildsten (2003) based on the WD temperature in DW UMa $(\approx 50,000 \mathrm{~K}$; AraujoBetancor et al. 2003; Knigge et al. 2000) assuming that the mass of the WD is in the range $0.6-1.0 M_{\odot}$.

However, the uncertainty with regard to the expected versus observed level of X-ray emission from DW UMa is exacerbated by the fact that at such a high mass transfer rate, the boundary layer has a temperature in excess of several hundred thousand degrees and is partially optically thick (e.g., Warner 1995, p. 67). This ensures that some fraction of the created (hard) $\mathrm{X}$-rays are self-absorbed at the source and do not contribute to the observed X-ray flux. On the other hand, this sets the stage for the scenario discussed for DW UMa at the start of this section: not only will immediate reprocessing of X-rays in the boundary layer result in a lower overall observed X-ray count rate, but also an X-ray spectrum that is skewed to low energies. Both of these effects are further exacerbated if the line of sight to the X-ray source passes through a large column density of an optically thick accretion disk. A nice corollary to this scenario is that, in addition to lowering the observed X-ray count rate and converting hard X-rays into soft, additional reprocessing in the disk can potentially explain the strong He II $\lambda 4686$ emission line observed in DW UMa and other SW Sex stars, via recombination following X-ray photoionization of the disk surface (Patterson \& Raymond 1985).

This leaves the question of the exact nature of the reprocessing/re-emission site that allows us to still see some $\mathrm{X}$-rays even during the eclipse. Our X-ray light curve of DW UMa is similar in behavior to its FUV 1154-1167 A light curve 
(Hoard et al. 2003). The FUV light curve does not sample a complete orbital cycle in phase (in particular, the eclipse phases were not observed-see Figure 5 in Hoard et al. 2003); however, the $\approx 65 \%$ phase coverage in the FUV light curve is consistent with the $\mathrm{X}$-ray light curve in which the flux decreases following $\phi=0.0$ until $\phi \approx 0.3$, then increases again toward $\phi=0.0$. In Hoard et al. (2003), we suggested that the FUV orbital modulation was caused by reprocessing of high energy luminosity originating from near the WD in a vertically extended structure visible above the self-occulting disk. The fact that the FUV and $\mathrm{X}$-ray light curves are modulated at all implies that the reprocessing site is asymmetric in some way; that is, it does not seem likely that it could be due to an accretion disk corona/wind unless that corona is non-uniform (e.g., in shape or density). See Hoard et al. (2003) for a detailed discussion of several possible vertically extended structures that could serve as the X-ray and FUV reprocessing site in DW UMa; briefly, these include a number of possible configurations of the accretion stream if it overflows its initial impact with the edge of the disk (also see discussion below), or vertical "curtains" produced at the truncated inner edge of the disk in a weakly magnetic WD (i.e., intermediate polar) stream-fed accretion scenario.

We also note that the Chandra X-ray light curve of the intermediate polar EX Hya (Hoogerwerf et al. 2005) bears some similarity to our XMM-Newton light curve of DW UMa. Both CVs show a narrow, partial X-ray eclipse, as well as a weak orbital modulation of the X-ray emission. In EX Hya, however, the X-rays are weakest at $\phi \approx 0.8$, which Hoogerwerf et al. (2005) interpret as being due to absorption in a bulge on the outer edge of the magnetically truncated accretion disk at the accretion stream impact site. In DW UMa, on the other hand, the brightest X-ray (and optical-UV) flux emerges at this orbital phase, suggesting the interesting possibility that the stream impact site itself could be a source of soft X-rays (or the primary site for reprocessing and re-emission of hard X-rays intercepted from the inner disk/WD region). This is consistent with the phase of weakest X-ray emission in DW UMa being located 0.5 orbital cycles later, when our line of sight would see only the back side of the stream-impact bulge (which is also largely occulted by the bulk of the disk at that phase).

Pratt et al. (2004) note that the column density for the hard component in their X-ray spectral fit for UX UMa is several orders of magnitude higher than for the soft component, consistent with viewing the hard X-ray source through the high inclination ( $i=70.2 \pm 0.2$; Linnell et al. 2008) disk in UX UMa. Based on comparison with UX UMa, we might expect a longer X-ray observation of DW UMa (in which a more statistically significant number of high energy $\mathrm{X}$-rays could be detected) to show a total eclipse of the high energy X-rays and require a larger column density to fit the hard component than the soft. However, the high energy count rate in UX UMa is $\approx 15 \%-20 \%$ of the total X-ray count rate (compared to $\lesssim 5 \%$ in DW UMa), so it is also possible that the disk in DW UMa is more optically thick and/or the $\approx 10^{\circ}$ difference in inclination between the two CVs produces a significant increase in column density along our line of sight through the disk in DW UMa. Thus, even a longer X-ray observation of DW UMa might not reveal a significant high energy X-ray count rate.

\section{CONCLUSIONS}

The X-ray spectrum of DW UMa can be fit equally well by a one-component cooling flow model, with a temperature range of $0.2-3.5 \mathrm{keV}$, or a two-component, two-temperature thermal plasma model, containing hard $(\sim 5-6 \mathrm{keV})$ and soft $(\sim 0.8 \mathrm{keV})$ components. Its X-ray light curve shows a likely partial eclipse, implying X-ray reprocessing in a vertically extended region, and an orbital modulation, implying a structural asymmetry in the X-ray reprocessing site (e.g., it cannot be a uniform corona). Regardless of the exact nature of the X-ray reprocessing site in DW UMa, the lack of a prominent hard X-ray total eclipse and very low fraction of high energy X-rays point to the presence of an optically and geometrically thick accretion disk that obscures the boundary layer and modifies the $\mathrm{X}$-ray spectrum emitted near the WD in this bright novalike CV and archetypal SW Sex star. This result continues to add credibility to the self-occulting disk model, which is supported by observational evidence spanning wavelengths from the optical through the FUV, and now to $\mathrm{X}$-rays.

This work was supported by an XMM-Newton Guest Observer grant for program 014297 and is based on observations obtained with XMM-Newton, a European Space Agency (ESA) science mission with instruments and contributions directly funded by ESA Member States and the United States of America (through the National Aeronautics and Space Administration). This work was performed, in part, at the Jet Propulsion Laboratory, California Institute of Technology. We utilized the SIMBAD database, operated at CDS, Strasbourg, France, and NASA's Astrophysics Data System.

\section{REFERENCES}

Andronov, N., Pinsonneault, M., \& Sills, A. 2003, ApJ, 582, 358

Araujo-Betancor, S., et al. 2003, ApJ, 583, 437

Arnaud, M., \& Raymond, J. 1992, ApJ, 398, 394

Arnaud, M., \& Rothenflug, M. 1985, A\&AS, 60, 425

Ballouz, R.-L., \& Sion, E. M. 2009, ApJ, 697, 1717

Baskill, D. S., Wheatley, P. J., \& Osborne, J. P. 2001, MNRAS, 328, 71

Baskill, D. S., Wheatley, P. J., \& Osborne, J. P. 2005, MNRAS, 357, 626

Beuermann, K., Thorstensen, J. R., Schwope, A. D., Ringwald, F. A., \& Sahin, H. 1992, A\&A, 256, 442

Buat-Ménard, V., Hameury, J.-M., \& Lasota, J.-P. 2001, A\&A, 366, 612

Dhillon, V. S., Marsh, T. R., \& Jones, D. H. P. 1991, MNRAS, 252, 342

Hellier, C., Kemp, J., Naylor, T., Bateson, F. M., Jones, A., Overbeek, D., Stubbings, R., \& Mukai, K. 2000, MNRAS, 313, 703

Hellier, C., Ringwald, F. A., \& Robinson, E. L. 1994, A\&A, 289, 148

Henden, A. A., \& Honeycutt, R. K. 1995, PASP, 107, 324

Hilton, E. J., Szkody, P., Mukadam, A., Henden, A., Dillon, W., \& Schmidt, G. D. 2009, AJ, 137, 3606

Hilton, E. J., Szkody, P., Mukadam, A., Mukai, K., Hellier, C., van Zyl, L., \& Homer, L. 2007, AJ, 134, 1503

Hoard, D. W., Szkody, P., Froning, C. S., Long, K. S., \& Knigge, C. 2003, AJ, 126,2473

Hoard, D. W., Thorstensen, J. R., \& Szkody, P. 2000, ApJ, 537, 936

Honeycutt, R. K., \& Kafka, S. 2004, AJ, 128, 1279

Honeycutt, R. K., Schlegel, E. M., \& Kaitchuck, R. H. 1986, ApJ, 302, 388

Hoogerwerf, R., Brickhouse, N. S., \& Mauche, C. W. 2005, ApJ, 628, 946

Jansen, F., et al. 2001, A\&A, 365, L1

Kaastra, J. S. 1992, An X-Ray Spectral Code for Optically Thin Plasmas, Internal SRON-Leiden Report, updated version 2.0

Kafka, S., \& Honeycutt, R. K. 2006, AJ, 132, 1517

Knigge, C., Araujo-Betancor, S., Gänsicke, B. T., Long, K. S., Szkody, P., Hoard, D. W., Hynes, R. I., \& Dhillon, V. S. 2004, ApJ, 615, L129

Knigge, C., Long, K. S., Hoard, D. W., Szkody, P., \& Dhillon, V. S. 2000, ApJ, 539, L49

Knigge, C., Long, K. S., Wade, R. A., Baptista, R., Horne, K., Hubeny, I., \& Rutten, R. G. M. 1998, ApJ, 499, 414

Lasota, J.-P. 2001, New Astron. Rev., 45, 449

Liedahl, D. A., Osterheld, A. L., \& Goldstein, W. H. 1995, ApJ, 438, L115

Linnell, A. P., Godon, P., Hubeny, I., Sion, E. M., \& Szkody, P. 2008, ApJ, 688, 568

Luna, G. J. M., Raymond, J. C., Brickhouse, N. S., Mauche, C. W., Proga, D., Steeghs, D., \& Hoogerwerf, R. 2010, ApJ, 711, 1333

Mason, K. O., et al. 2001, A\&A, 365, L36 
Mewe, R., Gronenschild, E. H. B. M., \& van den Oord, G. H. J. 1985, A\&AS, 62, 197

Mewe, R., Lemen, J. R., \& van den Oord, G. H. J. 1986, A\&AS, 65, 511

Mukai, K., Kinkhabwala, A., Peterson, J. R., Kahn, S. M., \& Paerels, F. 2003, ApJ, 586, L77

Mukai, K., Zietsman, E., \& Still, M. 2009, ApJ, 707, 652

Mushotzky, R. F., \& Szymkowiak, A. E. 1988, in NATO ASIC Proc. 229, Cooling Flows in Clusters and Galaxies, ed. A. C. Fabian (Dordrecht: Kluwer), 53

Nucita, A. A., Carpano, S., de Paolis, F., Ingrosso, G., Maiolo, B. M. T., \& Guainazzi, M. 2009a, New Astron., 14, 302

Nucita, A. A., Maiolo, B. M. T., Carpano, S., Belanger, G., Coia, D., Guainazzi, M., de Paolis, F., \& Ingrosso, G. 2009b, A\&A, 504, 973

Osaki, Y. 1996, PASP, 108, 39

Pandel, D., Córdova, F. A., \& Howell, S. B. 2003, MNRAS, 346, 1231

Pandel, D., Córdova, F. A., Mason, K. O., \& Priedhorsky, W. C. 2005, ApJ, 626, 396

Patterson, J., \& Raymond, J. C. 1985, ApJ, 292, 550

Popham, R., \& Narayan, R. 1995, ApJ, 442, 337

Pratt, G. W., Mukai, K., Hassall, B. J. M., Naylor, T., \& Wood, J. H. 2004, MNRAS, 348, L49

Ramsay, G., et al. 2001, A\&A, 365, L288

Rappaport, S., Verbunt, F., \& Joss, P. C. 1983, ApJ, 275, 713

Rodríguez-Gil, P., Casares, J., Dhillon, V. S., \& Martínez-Pais, I. G. 2000, A\&A, 355,181

Rodríguez-Gil, P., Casares, J., Martínez-Pais, I. G., Hakala, P., \& Steeghs, D. 2001, ApJ, 548, L49
Rodríguez-Gil, P., Martínez-Pais, I. G., \& de La Cruz Rodríguez, J. 2009, MNRAS, 395, 973

Rodríguez-Gil, P., Schmidtobreick, L., \& Gänsicke, B. T. 2007, MNRAS, 374, 1359

Shafter, A. W., Hessman, F. V., \& Zhang, E. H. 1988, ApJ, 327, 248

Smith, R. K., \& Brickhouse, N. S. 2000, RevMexAA Conf. Ser., 9, 134

Smith, R. K., Brickhouse, N. S., Liedahl, D. A., \& Raymond, J. C. 2001a, ApJ, 556, L91

Smith, R. K., Brickhouse, N. S., Liedahl, D. A., \& Raymond, J. C. 2001b, in ASP Conf. Ser. 247, Spectroscopic Challenges of Photoionized Plasmas, ed. G. Ferland \& D. W. Savin (San Francisco, CA: ASP), 159

Spruit, H. C., \& Ritter, H. 1983, A\&A, 124, 267

Stanishev, V., Kraicheva, Z., Boffin, H. M. J., Genkov, V., Papadaki, C., \& Carpano, S. 2004, A\&A, 416, 1057

Szkody, P., \& Piché, F. 1990, ApJ, 361, 235

Thorstensen, J. R., Ringwald, F. A., Wade, R. A., Schmidt, G. D., \& Norsworthy, J. E. 1991, AJ, 102, 272

Townsley, D. M., \& Bildsten, L. 2003, ApJ, 596, L227

Warner, B. 1986, MNRAS, 222, 11

Warner, B. 1995, Cataclysmic Variable Stars (Cambridge: Cambridge Univ. Press)

Wheatley, P. J., \& West, R. G. 2003, MNRAS, 345, 1009

White, N. E., Giommi, P., \& Angelini, L. 2000, VizieR Online Data Catalog, 9031, 0

Willems, B., Kolb, U., Sandquist, E. L., Taam, R. E., \& Dubus, G. 2005, ApJ, 635,1263

Zhang, E. 1988, Acta Astrophys. Sin., 8, 251 